\title{
SUPERNOVA REMNANT SHOCKS IN AN INHOMOGENEOUS INTERSTELLAR MEDIUM
}

\author{
Christopher F. McKee \\ Departments of Physics and of Astronomy \\ University of California, Berkeley CA 94720
}

\begin{abstract}
The inhomogeneity of the interstellar medium (ISM) has a profound effect on the propagation of the interstellar shock generated by a supernova and on the appearance of the resulting supernova remnant (SNR). Low mass supernovae produce remnants that interact with the "pristine" ISM, which has density inhomogeneities (clouds) on a wide range of scales. The shock compresses and accelerates the clouds it encounters; inside the blast wave, the clouds are hydrodynamically unstable, and mass is injected from the clouds into the intercloud medium. Embedded clouds interact thermally with the shock also, adding mass to the hot intercloud medium via thermal evaporation or subtracting it via condensation and thermal instability. Mass injection into the hot intercloud medium, whether dynamical or thermal, leads to infrared emission as dust mixes with the hot gas and is thermally sputtered. The remnants of massive supernovae interact primarily with circumstellar matter and with interstellar material which has been processed by the ionizing radiation and wind of the progenitor star. After passing through any circumstellar material which may be present, the shock encounters a cavity which tends to "muffle" the SNR. The remnants of massive supernovae therefore tell us more about the late stages of the evolution of massive stars than about the ISM.
\end{abstract}

\section{INTRODUCTION}

The ISM is observed to be highly inhomogeneous: most of the mass is concentrated in diffuse atomic clouds $(T \sim 100 \mathrm{~K})$ and dense molecular clouds $(T \sim 10 \mathrm{~K})$ which occupy a small fraction of the volume, whereas much of the volume is occupied by low density, warm $\mathrm{HI}$ and $\mathrm{HII}\left(T \sim 10^{4} \mathrm{~K}\right)$ and by very low density, hot coronal gas $\left(T \sim 10^{6} \mathrm{~K}\right)$. This inhomogeneity is a direct consequence of the the energy flow from stars through the thermally unstable ISM and into the intergalactic medium (McKee 1986). If the rate of stellar energy injection were very low, then the ISM would settle into a thin, quiescent HI disk; in order to be stable against gravitational collapse, the mean density of the medium would have to be low. At the opposite extreme of a high energy injection rate, the ISM would heat up to $T \gg 10^{6} \mathrm{~K}$ and blow away in a galactic wind. For a broad range of intermediate energy injection rates, the ISM settles into a multiphase medium such as we observe (Ikeuchi, Habe, and Tanaka 1984).

Stars inject energy into the ISM in the form of radiation, supernova explosions, and winds. By far the dominant constituent of this energy flow is starlight. Most of this radiation escapes into the intergalactic medium without interacting with the interstellar gas, although it may heat interstellar dust en route. A fraction of the far-ultraviolet radiation between about $6 \mathrm{eV}$ and the ionization limit of hydrogen at $13.6 \mathrm{eV}$ heats the gas by ejecting photoelectrons 
from dust grains; this may well be the dominant heating mechanism for the HI, and it leads naturally to a two-phase medium with $100 \mathrm{~K}$ clouds embedded in a $10^{4} \mathrm{~K}$ intercloud medium (deJong 1980). Ionizing stellar radiation $(h \nu>13.6$ $\mathrm{eV}$ ) is quite effective at heating the gas. Its effects tend to be localized in HII regions, but it has a profound effect on the evolution and appearance of the SNRs which occur there.

After starlight, the most important contributor to the stellar energy flux is supernova explosions. Supernovae are a particularly important form of energy injection because they probably determine the velocity distribution of interstellar clouds (e.g., Spitzer 1968a), they are responsible for the acceleration of cosmic rays, and they can produce large volumes of hot coronal gas (Cox and Smith 1974). If the filling factor $f_{h}$ of the hot gas is large $\left(f_{h} \gtrsim \frac{1}{2}\right)$, the ISM is a three phase medium with cold and warm clouds embedded in the hot gas; SNRs expand primarily in the hot gas and determine the pressure of the ISM (McKee and Ostriker 1977). Although $f_{h}$ is high in the local ISM, its value at a typical point in the galactic disk remains controversial (Cox 1987). It is quite likely that $f_{h}$ depends on both the nature of the galaxy and the location within it. In principle, observations of SNRs could provide the means to measure $f_{h}$, but it would first be necessary to determine which SNRs are interacting with the "pristine" ISM, unaffected by the progenitor star. As we shall see below, this is not a straightforward task.

Energy injection via stellar winds is negligible for low mass stars. For massive stars, the total wind energy injection during core hydrogen burning is less than that in the final supernova explosion unless the the star is initially very massive, $M \gtrsim 60 M_{\odot}$ (Abbott 1982). Stars initially above about $40 M_{\odot}$ are believed to go through a Wolf-Rayet stage after exhausting their central hydrogen (Chiosi and Maeder 1986), during which the wind energy injection is comparable to that of a supernova (Abbott 1982). Comparing the total supernova energy injection with the total wind energy injection in the Galaxy, Abbott (1982) concluded that supernovae dominate by a factor of about five. Winds do have two important effects, however: First, the interstellar bubbles of hot, low density gas produced by these winds (Castor, McCray, and Weaver 1975) "muffle" the final supernova explosion, making the resulting SNR more difficult to detect. Second, a significant amount of mass can be ejected by low velocity winds, which are energetically unimportant but observationally critical, since the interaction of the SNR blast wave with this circumstellar matter is readily observable.

The flow of stellar energy through the ISM thus leads to two qualitatively different types of inhomogeneity: In the general ISM, the medium is characterized by clouds embedded in a lower density intercloud medium, whereas near massive stars the ionizing radiation and winds reconfigure the ambient ISM into a radially stratified medium. Supernovae from low mass stars (Type Ia) explode in the pristine ISM and should provide a powerful probe of its structure. Supernovae from massive stars (Type II and, presumably, Type Ib--e.g., Wheeler and Levreault 1985) cannot "see" the pristine ISM until they have expanded to very large sizes and become difficult to observe. Here we shall explore the consequences of both types of inhomogeneity on the structure and appearance of an SNR, and how SNRs in turn affect the ambient medium. 


\section{SNR SHOCKS IN A CLOUDY ISM}

\subsection{Cloud Crushing}

An SNR in a cloudy medium expands through the intercloud medium, driving shocks into the clouds it overtakes. There is a simple relation between the velocity of the cloud shock, $v_{s}$, and that of the SNR blast wave, $v_{b}$. Let $\rho_{i 0}$ be the initial density of the intercloud medium, and let $\rho_{c 0}$ be the density of the cloud. We assume that the shocks are strong: the pressure behind the shocks greatly exceeds that ahead, so that they are highly supersonic. Then the pressure behind the blast wave shock is $\sim \rho_{i 0} v_{b}^{2}$, and that behind the cloud shock is $\sim \rho_{c 0} v_{s}^{2}$. Since the pressure of the shocked intercloud gas drives the shock into the cloud, these pressures must be comparable, and we conclude that (Bychkov and Pikel'ner 1975, McKee and Cowie 1975)

$$
v_{s} \simeq\left(\rho_{i 0} / \rho_{c 0}\right)^{1 / 2} v_{b} .
$$

A more precise evaluation, which allows for the decrease in $v_{s}$ and $v_{b}$ with time as the SNR expands, shows that for a Sedov-Taylor blast wave this simple result should be accurate to within a factor of about 1.5 ( $c f$. McKee et al. 1987). However, an important caveat should be kept in mind: the derivation is based on the assumption that the blast wave is non-radiative, so that the ram pressure and the thermal pressure behind the blast wave shock are comparable. If, on the other hand, the blast wave is radiative, then the cloud shock velocity is $v_{s} \simeq\left(\rho_{i 1} / \rho_{c 0}\right)^{1 / 2} v_{b}$, where $\rho_{i 1}$, the density of of the shocked intercloud medium, may be up to 100 times greater than $\rho_{i 0}$. We focus on non-radiative blast waves here.

The fate of a cloud engulfed by a blast wave depends on its size. For simplicity, assume that the cloud is approximately spherical, with radius $a$. Let $R$ be the distance between the cloud center and the site of the $\mathrm{SN}$ explosion. The blast wave expands as $R_{b} \propto t^{\eta}$, where $\eta=2 / 5$ for a Sedov-Taylor blast wave. Let

$$
\chi \equiv \frac{\rho_{c 0}}{\rho_{i 0}}
$$

be the cloud/intercloud density ratio; we assume $\chi \gg 1$. The three relevant time scales are the cloud crushing time,

$$
t_{c c} \equiv \frac{a}{v_{s}}=\frac{\chi^{1 / 2} a}{v_{b}}
$$

the intercloud crossing time,

$$
t_{i c} \equiv \frac{2 a}{v_{b}}
$$

and the age of the SNR,

$$
t \equiv \frac{\eta R}{v_{b}}=\frac{2}{5} \frac{R}{v_{b}},
$$

where the second equality in equation (5) is for Sedov-Taylor blast waves. The use of $a$ rather than $2 a$ in the definition of the cloud crushing time $t_{c c}$ is rather arbitrary, but it does allow for the fact that the cloud is crushed from both sides. 
Our assumption that the density ratio $\chi$ is much greater than 1 ensures that $t_{c c}>t_{i c}$. Clouds then come in one of three sizes, small, medium, or large:

* Small clouds: $\quad t>t_{c c} \Longrightarrow a<\eta R / \chi^{1 / 2}=0.4 R / \chi^{1 / 2}$. The SNR does not evolve significantly during the cloud crushing. For $t \gg$ $t_{c c}$, the cloud crushing is completed within a short distance behind the blast wave shock. Because the cloud is crushed promptly, the pressure driving the cloud shock, and hence $v_{s}$, are approximately constant.

* Medium clouds: $t_{c c}>t>t_{i c} \Longrightarrow \eta R / \chi^{1 / 2}<a<\eta R / 2$. The SNR does not evolve significantly while the blast wave in the intercloud medium crosses the cloud, but it does evolve while the cloud is being crushed. Since the pressure driving the cloud shock drops as the SNR expands, the cloud shock decelerates.

* Large clouds: $\quad t_{i c}>t \Longrightarrow a>\eta R / 2=0.2 R$. The blast wave weakens significantly between the time it first encounters the cloud and the time it has crossed the cloud; since $R_{b}$ increases by at least a factor $1.2 / 0.8=1.5$ in crossing a large cloud, the blast wave pressure $P \propto R_{b}^{-3}$ drops by at least a factor 3 . The force exerted on the cloud is impulsive, so the cloud shock decelerates significantly. If the cloud is somewhat larger than the minimum large cloud, it will affect the overall expansion of the blast wave; a cloud with $a>0.5 R$ occupies more than about $1 / 16$ of the volume of the SNR.

In sum, the crushing of a small cloud is like being squeezed by a vise, whereas that of a large cloud is like being struck by a hammer.

Cloud crushing occurs in several well-defined stages ( $c f$. Nittman, Falle, and Gaskell 1982; Heathcote and Brand 1983). We shall focus on small clouds, since most of the theoretical work has concentrated on this case.

i) Initial transient. When the blast wave first strikes the cloud, a transmitted shock propagates into the cloud and a reflected shock propagates back upstream into the shocked intercloud gas. So long as these shocks are approximately plane parallel, the pressure between them is about 6 times that behind the initial blast wave shock (for a $\gamma=5 / 3$ gas and $\chi \gg 1$ : Silk and Solinger 1973). In a time of order $a / v_{b}=t_{i c} / 2$, the reflected shock in the intercloud medium settles into a standing bow shock. The peak pressure between the shocks is then only about 3 times that behind the initial blast wave shock (McKee and Cowie 1975).

ii) Shock compression. After a time of order $t_{i c}$, the flow around the cloud converges on the axis behind the cloud, producing a reflected shock in the intercloud gas and driving a shock into the rear of the cloud (Woodward 1976). The shocks compressing the cloud from the sides are weaker than those compressing it fore and aft because the pressure in the flow around an obstacle has a minimum at the sides (Nittman et al. 1982). The result is that the cloud is crushed into a thin pancake, with its lateral dimensions reduced by about a factor 2 ; the main cloud shock propagating in from the nose of the cloud is stronger than the secondary shock propagating in from the rear 
(Woodward 1976). If the cloud shock is fast enough $\left(v_{s} \gtrsim 200 \mathrm{~km}\right.$ $\left.\mathrm{s}^{-1}\right)$ it will be non-radiative; otherwise it will be radiative and the cloud will be compressed to high density (Sgro 1975). The collision of the main and secondary shocks results in yet greater compression.

iii) Re-expansion. When the main cloud shock reaches the rear of the cloud, a strong rarefaction is reflected back into the cloud, causing the cloud to expand downstream (Woodward 1976); the low pressure at the sides of the cloud induces it to expand sideways also (Nittman et al. 1982).

iv) Final fate. Nittman et al. (1982) and Heathcote and Brand (1983) have suggested that hydrodynamic instabilities will subsequently destroy the cloud; Nittman et al. also invoked the lateral expansion of the cloud as a destruction mechanism. The possible stabilizing effects of magnetic fields have yet to be considered. Even if the cloud did break up into a spray of very small cloudlets as a result of instabilities, the spray might behave as a cloud until either thermal evaporation caused it to merge with the intercloud medium, or expansion reduced its mean density to that of the intercloud medium. The final fate of a small cloud in a SNR thus remains an open question; medium and large clouds are unlikely to be destroyed.

The net acceleration of the cloud by the blast wave is due to two effects, shock acceleration and ram pressure acceleration (McKee, Cowie, and Ostriker 1978). The main cloud shock accelerates the gas up to $v_{1}=(3 / 4,1) v_{\text {s }}$ if the shock is (non-radiative, radiative), respectively; the average velocity of the entire cloud is slightly less than this because of the secondary shock propagating in from the the rear of the cloud. The ram pressure acceleration is due to the flow of intercloud gas past the cloud, which exerts a drag on the cloud. In the absence of magnetic fields, the drag for subsonic flow depends only on the Reynolds number $R e$ of the flow. Using Draine and Giuliani's (1984) value for the viscosity $\nu$ of an ionized plasma of cosmic abundance, we have

$$
R e=\frac{a v_{i}}{\nu}=15.8 \frac{n_{i} \mathcal{M} a_{\mathrm{pc}}}{T_{i 7}^{2}},
$$

where $\mathcal{M} \equiv v_{i} / C_{i}=v_{i}\left(\rho_{i} / P_{i}\right)^{1 / 2}$ is the isothermal Mach number of the intercloud gas, $n_{i}$ is the density of hydrogen nuclei in the intercloud gas, $T_{i 7} \equiv T_{i} / 10^{7} \mathrm{~K}$, and $a_{\mathrm{pc}}$ is the cloud radius in parsecs. In terms of the proton-proton mean free path $\lambda_{p}=6.00 \times 10^{17} T_{i 7}^{2} / n_{i} \mathrm{~cm}$ (see Spitzer 1962), this is

$$
R e=3.07\left(a / \lambda_{p}\right) \mathcal{M}
$$

Hence, in the absence of magnetic fields, the ion mean free path inside an SNR is quite long and the flow can be quite viscous. Magnetic fields reduce the mean free path by many orders of magnitude, however, with a corresponding reduction in the viscosity and increase in the Reynolds number: for typical conditions in the intercloud medium $\left(n_{i}=0.2 \mathrm{~cm}^{-3}, B=3 \mu \mathrm{G}\right)$, the Reynolds number perpendicular to the magnetic field increases by a factor $2 \times 10^{19} T_{i 7}^{3}$ ! (For a general discussion of viscosity in a magnetized plasma, focusing on viscous heating, 
see Hollweg [1986].) Although this reduction in the viscosity occurs only perpendicular to the field, the full effect of the reduction is likely to occur in the problem at hand because the flow of the gas past the cloud will tend to drape the field lines along the boundary layer between the cloud and intercloud medium. If the magnetic field in the boundary layer becomes dynamically important, it could suppress the turbulence which sets in there for $R e \gtrsim 10^{5}$ in the absence of a field. For flow past a sphere, the drag coefficient $C_{D}$ (the ratio of the drag to $0.5 \rho_{i} v_{i}^{2} \pi a^{2}$ ) is about 0.4 for $R e \sim 10^{5}$; for flow past a circular disk, similar to the configuration the cloud reaches near the end of the shock compression phase, $C_{D} \simeq 1.1$ over a wide range of $R e$ (Batchelor 1967). For supersonic flow, such as we are considering here, the drag is greater because of the emission of sound waves; for flow past a sphere, $C_{D} \sim 1.0$ (Chernyi 1961), and for flow past a plate $C_{D}$ is somewhat larger still. McKee et al. (1978) adopted $C_{D}=2$. In terms of the nomenclature introduced here, they found that small clouds are strongly affected by ram pressure acceleration, whereas medium and large clouds are not. Nittman et al. (1982) have argued that ram pressure acceleration is unimportant because the lateral expansion of the cloud will destroy it before substantial acceleration can occur. Their argument implicitly assumes that the cloud shock is non-radiative, however, and does not apply to the more common case of radiative cloud shocks. Furthermore, even in the case of non-radiative cloud shocks, substantial ram pressure acceleration can occur as the lateral expansion reduces the mass per unit area of the cloud, making it more like a sail than a cannonball. Medium and large clouds are accelerated primarily by the cloud shock, which decelerates as it propagates through the cloud. The dynamics of the decelerating cloud shock, and the pressure and velocity distribution behind it, have been discussed by McKee et al. (1987); at late times, the cloud shock is approximately momentum-conserving. Because the blast wave also decelerates, the medium and large clouds can coast beyond the blast wave shock at late times, an effect dramatically portrayed in the numerical simulations of Tenorio-Tagle and Rozcyzka (1986) and Rozcyzka and Tenorio-Tagle (1987).

The interaction of the blast wave with a cloud is subject to both Rayleigh-Taylor and Kelvin-Helmholtz instabilities. When the blast wave first strikes the cloud, it subjects the dense cloud gas to an instantaneous acceleration by the light intercloud gas. This results in a transient Rayleigh-Taylor instability which produces a variation in the velocity of the interface of $\delta v \simeq\left(k a_{0}\right) v_{1}$ for $\chi \gg 1$, where $a_{0}$ is the initial amplitude of the displacement of the interface, $k$ is the wavenumber of the displacement, and $v_{1}$ is the velocity of the shocked cloud gas (Richtmyer 1960). The derivation of this result assumes that $k a_{0} \ll 1$; hence, the velocity variation $\delta v$ is small compared to the velocity $v_{1}$ imparted to the cloud gas, and the instability significantly alters the shape of the cloud only after the cloud has undergone substantial compression. For a planar cloud, no further instability would result. However, the finite size of the cloud leads to a convergence and acceleration of the cloud shock, and hence a further weak Rayleigh-Taylor instability (Woodward 1976). The Kelvin-Helmholtz instability due to the flow of the intercloud gas past the cloud strongly distorts the surface of the cloud and can ultimately lead to substantial ablation of the cloud (Nulsen 1982; see below).

Because of the complex nonlinear nature of the blast wave-cloud interaction, it appears that only numerical simulation can determine the ultimate fate of the cloud: What is the total momentum transferred to the cloud? How 
much mass is lost from the cloud? Is the cloud disrupted, distorted, or driven into gravitational collapse? What is the effect of the interstellar magnetic field? To date, no conclusive answer is available to any of these questions. Even if we assume that the magnetic field is dynamically unimportant, there are two major problems which have tended to confound efforts at numerical simulation. The first and most obvious problem is numerical diffusion at the cloud-intercloud interface. Remarkably, the only calculation which overcomes this problem was published over a decade ago, by Woodward (1976; see also Woodward 1979). He used a code which had an Eulerian treatment of the intercloud medium and a Lagrangian treatment of the cloud, thereby permitting accurate treatment of vorticity in the intercloud gas and of the radiative shock in the cloud. The problem he addressed was the collision of a density wave shock with a cloud; since the pressure behind the shock was time-independent, the cloud was effectively "small". The pressure jump was only a factor 8 , considerably weaker than in a typical SNR shock; as a result, the flow behind the shock was subsonic relative to the cloud and a bow shock did not form. He found that most of the cloud was shocked by the main cloud shock, resulting in a pancake-shaped cloud near the end of the compression phase. The cloud was strongly distorted by Kelvin-Helmholtz and Rayleigh-Taylor instabilities, and the effort involved in manually rezoning the interface forced him to terminate the calculation before the end of the compression phase. Subsequent calculations (e.g., Nittman et al. 1982, Tenorio-Tagle and Rozcyzka 1986, Rozcyzka and Tenorio-Tagle 1987) appear to have strong numerical diffusion at the boundary, making it difficult to disentangle cloud/intercloud mixing due to instabilities from mixing due to numerical effects.

The second problem confronting the numerical simulator is the disparity between the actual Reynolds number of the flow and the effective Reynolds number in the simulation. For example, Woodward's (1976) simulation had a grid size of $0.25 \mathrm{pc}$ in the intercloud gas; if this is taken to be the effective mean free path, then for $\mathcal{M} \sim 1$ we have $R e \sim 180$ from equation (7). By contrast, in the absence of a magnetic field, the proton mean free path is about $3 \times 10^{13} \mathrm{~cm}$, giving $R e \sim 5 \times 10^{6}$. If this were a simulation of non-magnetic, subsonic flow past a rigid sphere, the discrepancy in $R e$ would be significant because the boundary layer and wake would be turbulent in the physical case and laminar in the simulation, so that, for example, the drag in the physical case would be about half that in the model (Batchelor 1967). In fact, however, it was a simulation of nearly sonic flow past a sphere roughened by instabilities; the interstellar magnetic field would probably not have been dynamically important, but it would have increased the Reynolds number perpendicular to the field by about 10 orders of magnitude. My own feeling is that the Reynolds number disparity will not significantly limit our ability to determine the final fate of a blasted cloud, although it will limit our ability to follow the details of the flow in the intercloud medium.

One of the major motivations for studying the blast wave-cloud interaction has been to determine the conditions under which shock compression can induce gravitational collapse. Woodward (1976) found that dense globules formed by the combined action of the Kelvin-Helmholtz and Rayleigh-Taylor instabilities became gravitationally bound and presumably would have formed stars, although an unrealistically low magnetic field $(B<1 \mu \mathrm{G})$ would have been required. Nittman (1981) was the first to include a magnetic field in the simulation. He demonstrated that a dynamically important field would not only limit the density reached by the shocked cloud, but also reduce the shear which led to the Kelvin-Helmholtz in the first place. A more direct method of driving 
gravitational collapse, and one less subject to numerical difficulties, has been studied by Hillebrandt and his collaborators: namely, to compress the entire cloud to the point of instability. Krebs and Hillebrandt (1983) considered large clouds ( $a=10 \mathrm{pc}, R=30 \mathrm{pc}$ ) initially close to the maximum stable mass $M_{J}$; indeed, their Model A started with $M>M_{J}$ (the ratio of central to mean density was about 10, larger than the maximum value of 5.78 allowed for stable configurations [Spitzer 1968b]). The remaining two models they considered had $M \sim 0.4 M_{J}$, and they found that a $10^{51} \mathrm{erg}$ supernova induced gravitational collapse, whereas a $10^{50} \mathrm{erg}$ one did not. Subsequently, Oettl, Hillebrandt, and Müller (1985) studied the stabilizing effects of a magnetic field on Model $\mathrm{A}$.

\subsection{Mass Exchange}

If most of the mass of the interstellar medium is tied up in clouds, processes which transfer this mass into the intercloud medium can have a dramatic effect on the dynamics and appearance of an SNR blast wave. Correspondingly, processes which transfer mass from the intercloud medium back into the clouds, which tend to act when the blast wave becomes weak, are important in maintaining the multi-phase medium.

In this review we shall focus on mass exchange driven by thermal conduction. Inside the blast wave the temperature ratio of the shocked intercloud medium and the shocked clouds is of order $\chi \gg 1$ if the cloud shocks are nonradiative, and larger yet if they are radiative. Thermal conduction will act to smooth out the resulting sharp temperature gradients. How it does so depends on the ratio of the electron mean free path to the cloud size and on the topology of the magnetic field. For the moment we neglect the magnetic field, returning to its effects below. The effects of a finite mean free path can be measured in terms of the saturation parameter

$$
\sigma_{0}^{\prime} \equiv \frac{2}{25} \frac{\kappa_{i} T_{i}}{\rho_{i} C_{i}^{3} a}=1.67 \frac{\lambda_{e e}}{a}=0.393 \frac{T_{i 7}^{2}}{n_{i} a_{\mathrm{pc}}}
$$

(Cowie and McKee 1977, Balbus and McKee 1982), where $\kappa_{i}=5.6 \times 10^{-7} T_{i}^{5 / 2}$ erg s${ }^{-1} \mathrm{~K}^{-1} \mathrm{~cm}^{-1}$ is the classical thermal conductivity for a dilute plasma of cosmic abundance (Draine and Giuliani 1984) and $\lambda_{e e}$ is the mean free path for electronelectron energy exchange. The saturation parameter is inversely proportional to the Reynolds number: Recall that the Prandtl number measures the relative importance of viscosity and conduction, and for an ideal gas it is

$$
\operatorname{Pr}=\frac{5}{2} \frac{k \nu \rho}{\kappa \mu}
$$

(Landau and Lifschitz 1959). Neutral gases have $\operatorname{Pr} \sim 1$, but for an ionized cosmic plasma $P r=0.0321$ is small because the heat is carried by the electrons whereas the viscosity is due to the slower ions. Combining equations (6), (8), and (9) then shows that $\sigma_{0}^{\prime}$ is inversely proportional to $R e$ :

$$
\sigma_{0}^{\prime} R e=\frac{\mathcal{M}}{5 P r}=6.23 \mathcal{M}
$$


Provided radiative losses are negligible, the heat flux from the intercloud medium into the cloud heats the cloud surface and drives an evaporative flow away from the cloud. For $\sigma_{0}^{\prime} \lesssim 1$, thermal conduction is approximately described by classical theory with the heat flux $=\kappa \nabla T$. Balancing the heat flux $\kappa_{i} T_{i} / a$ against the energy flux $5 P v / 2$ gives an evaporation rate $\dot{m}=4 \pi a^{2} \rho v \sim 8 \pi a \kappa \mu / 5 a$ for spherical clouds. More precise evaluation gives

$$
\dot{m}=\frac{16 \pi \mu \kappa a}{25 k}=2.50 \times 10^{4} T_{i}^{5 / 2} a_{\mathrm{pc}} \quad g s^{-1}
$$

for $0.03 \lesssim \sigma_{0}^{\prime} \lesssim 1$ (Cowie and McKee 1977; the numerical coefficient is slightly lower than given there because we have used Draine and Giuliani's [1984] value for $\kappa$ ). The generalization to ellipsoidal clouds, including disks and needles, was obtained by Cowie and Songaila (1977). Balbus (1985) has further extended the theory to cover clouds of arbitrary shape and to cover systems of clouds. It must be emphasized that steady evaporative flow is possible only in 3 dimensions; in the planar case, the temperature gradient relaxes until radiative losses become important and the evaporative flow switches over to a cooling flow of the type considered by Doroshkevich and Zel'dovich (1981).

In general, we can write

$$
\dot{m}=\rho_{i} C_{i} A_{c} F\left(\sigma_{0}^{\prime}\right),
$$

where $A_{c}$ is the surface area of the cloud and $F$ is a number of order unity; for classical evaporation of spherical clouds, $F=2 \sigma_{0}^{\prime}$. If we define an ablation time $t_{a} \equiv m / \dot{m}$, then just behind the blast wave shock $\left(\rho_{i}=4 \rho_{i 0}, C_{i}^{2}=3 v_{b}^{2} / 16\right)$ we have

$$
\frac{t_{a}}{t_{c c}}=\frac{\chi^{1 / 2}}{3^{3 / 2} F}
$$

for a density ratio $\chi \sim 100$, clouds are crushed before they are ablated if $F \lesssim 2$.

When the electron mean free path becomes comparable to the cloud radius, the classical theory of heat conduction breaks down and heat transport becomes non-local: the heat flux at a given point depends not only on the temperature in the immediate neighborhood but also on the global temperature structure, since electrons have sufficiently long mean free paths to reach the point from substantial distances (e.g., Luciani, Mora, and Pellat 1985). Nonetheless, it is possible to develop a simple phenomenological model that captures the essential physics: In the limit of a very steep temperature gradient, collisions are negligible and the heat flux $q$ should saturate at a value proportional to that which would result from all the electrons streaming freely down the temperature gradient,

$$
q_{\mathrm{sat}}=f n_{e} k T_{e}\left(k T_{e} / m_{e}\right)^{1 / 2},
$$

where the "flux limit" $f$ is a numerical factor to be determined. The actual heat flux is then taken as the minimum of the classical and saturated values, or, if a smoother estimate is desired, as the harmonic mean,

$$
q=\frac{q_{\mathrm{cl}}}{1+\left(q_{\mathrm{cl}} / q_{\mathrm{sat}}\right)}
$$


where $q_{\mathrm{cl}}$ is the classical heat flux (Balbus and McKee 1982, Giuliani 1984). In evaporative flows, the maximum value of $q_{\mathrm{cl}} / q_{\mathrm{sat}}$ is comparable to $\sigma_{0}^{\prime}$. Cowie and McKee (1977) argued that $f \simeq 0.32$. Laser fusion experiments are consistent with a somewhat smaller value, $f \sim 0.1$ (see Max, McKee, and Mead 1980 and references therein). Recent analytic theory which allows for the streaming of suprathermal electrons (Campbell 1984) agrees with equation (15) to within a factor 1.4 with $f=0.32$; if attention is restricted to weakly saturated flows, $0.8 \gtrsim q / q_{\mathrm{cl}} \gtrsim 0.4$, the agreement improves with $f=0.15$. Campbell suggests that plasma instabilities could reduce $q$ by up to a factor 2 in the highly saturated case. The saturated heat flux can be expressed in terms of hydrodynamic variables as $q_{\mathrm{sat}}=5 \phi_{s} \rho C^{3}$, where $\phi_{s}=3.5 f$ for an ionized cosmic plasma. The range $0.3 \gtrsim f \gtrsim 0.1$ then corresponds to $1 \gtrsim \phi_{s} \gtrsim 0.3$. Cowie and McKee (1977) solved for the evaporation rate allowing for saturation of the heat flux in the range $100 \gtrsim \sigma_{0}^{\prime} \gtrsim 1$; for higher $\sigma_{0}^{\prime}$ the theory breaks down because the hot electrons can penetrate all the way to the cloud surface. In this range the factor $F\left(\sigma_{0}^{\prime}\right)$ in equation (12) is a slowly increasing function of $\sigma_{0}^{\prime}$, reaching a maximum of $(4,15)$ for $\phi_{\mathrm{s}}=(0.3,1)$. Numerical integrations by Giuliani $(1984)$ confirmed the analytic calculations of Cowie and McKee, the primary difference being the use of equation (15) by Giuliani instead of the $\operatorname{simpler} \min \left(q_{\mathrm{cl}}, q_{\mathrm{sat}}\right)$ used by Cowie and McKee. He and Draine and Giuliani (1984) also explored the effects of viscosity on evaporative flows. Saturation of the heat flux is important in the study of solar flares as well (e.g., Smith 1986).

When the electron mean free path becomes so large that the electrons can easily penetrate into the cloud, the uncertainty in the theory of saturated thermal conduction becomes less important. Such suprathermal evaporation is as though the cloud were bathed in a gas of cosmic rays; the cloud pressure is determined by the evaporative outflow and by the requirement that the cloud itself be in thermal balance with cosmic ray heating (Balbus and McKee 1982). This regime corresponds to $\sigma_{0}^{\prime} \gtrsim 10^{3}$, and values of $F$ up to $10^{3}$ are possible.

In the opposite limit of small mean free path, radiative losses become important. The condition that radiative losses balance conductive heating is $n^{2} \Lambda(T) \sim \nabla \cdot \kappa \nabla T$, where $\Lambda(T)$ is the cooling function; this defines a length scale

$$
\lambda_{F} \equiv\left(\frac{\kappa T}{n^{2} \Lambda}\right)^{1 / 2}
$$

For clouds small compared to $\lambda_{F}$, evaporation is unaffected by radiative losses, whereas for clouds large compared to $\lambda_{F}$, radiative losses dominate and gas condenses onto the clouds from the intercloud medium. The dividing line between evaporation and condensation occurs at a cloud radius $a \simeq 0.3 \lambda_{F}$ (McKee and Cowie 1977). In the temperature range $10^{7.5} \gtrsim T \gtrsim 10^{5} \mathrm{~K}$ the cooling function varies approximately as $T^{-1 / 2}$, so that this condition defines a critical value of the saturation parameter, $\sigma_{0}^{\prime} \simeq 0.03$. Classical evaporation is thus restricted to the range $1 \gtrsim \sigma_{0}^{\prime} \gtrsim 0.03$, and condensation occurs for smaller values of $\sigma_{0}^{\prime}$. Calculations of the non-equilibrium ionization in evaporative flows have been made by Ballet, Arnaud, and Rothenflug (1986), and self-consistent calculations of the dynamics of evaporative flows including radiative losses have been carried out 
recently by Böhringer and Hartquist (1987). Relatively little work has been done on condensation (Graham and Langer 1973, Doroshkevich and Zel'dovich 1981, Begelman and McKee 1987).

Magnetic fields can, in principle, reduce thermal conduction by an enormous factor, just as in the case of viscosity. There is an important distinction between the two, however: heat can be conducted along field lines even when they are dynamically unimportant, but viscous stress cannot. For evaporative flows in a stationary medium, Cowie and McKee (1977) stated that magnetic fields would have relatively little local effect on the evaporation rate; this was based on the idea that the outflow would comb out the field so that the heat from the external medium could more easily reach the cloud surface, and Balbus (1986) has recently confirmed this. However, blasted clouds are embedded in a moving ambient medium which draws the field out behind the cloud, significantly reducing the heat flux to the cloud (although this is partly compensated by the resulting increased temperature gradient). Magnetic reconnection should eventually allow the cloud to come back into thermal contact with its surroundings, and once the flow velocity past the cloud falls below the evaporative velocity (which is of order $\sigma_{0}^{\prime} C_{i}$ for classical evaporation), normal evaporation could resume. The global effects of magnetic fields on evaporation remain to be determined. Balbus (1986) argues that each cloud is in a one-dimensional flux tube, so that evaporation will cease once the temperature relaxes to the point that radiative losses become important. A more general way of looking at the problem is to consider each cloud to occupy a "basin" defined by the condition that heat flows toward the cloud in its basin (Begelman and McKee 1987). In the absence of a magnetic field, the size of the basin is of order the mean intercloud separation. With a field, the shape of the basin will be altered; the rate of evaporation will be reduced insofar as the mean distance to a point in the basin is increased, vanishing altogether when this distance becomes comparable to $\lambda_{F}$.

Hydrodynamic instabilities, particularly the Kelvin-Helmholtz instability, also lead to mass exchange between clouds and the intercloud medium. Nulsen (1982) has argued that the instability will saturate when the rate at which mass is stripped from the cloud is large enough to smooth out the velocity gradient driving the instability, which will occur when the momentum flux of the stripped gas is comparable to that impinging on the cloud. This gives a mass loss rate of order $\dot{m}=\pi a^{2} \rho_{i} v_{i}$, corresponding to $F=\mathcal{M} / 4$ in equation (12). He terms this process "turbulent viscous stripping." This process is effective when evaporation is not: it is independent of the orientation of the magnetic field, so long as the field is weak; it works best when the relative velocity is high (but subsonic relative to the intercloud gas); and, since $\dot{m} \propto T^{1 / 2}$ rather than $T^{5 / 2}$, it is relatively more important at moderate temperatures. Pieces of the cloud torn off by the instability will eventually merge with the intercloud medium by thermal evaporation once they are comoving and become magnetically connected with the intercloud medium.

This discussion has concentrated on the processes by which mass is transferred from clouds into the intercloud medium. The reverse process occurs by radiative cooling. For hot gas with $\Lambda=1.6 \times 10^{-19} T^{-1 / 2} \mathrm{erg} \mathrm{cm}^{3} \mathrm{~s}^{-1}$, the cooling time is proportional to the entropy variable $s \equiv T^{3 / 2} / n$ :

$$
t_{\text {cool }}=6.3 \times 10^{-5} s \quad \text { yr }
$$


(Kahn 1976, McKee 1982), which is independent of any expansion or contraction the gas experiences. Mass injection into the intercloud medium reduces $s$ and hence the cooling time. The cooling gas can condense onto pre-existing clouds, or, because it is thermally unstable (Field 1965; McCray, Stein, and Kafatos 1975) it can form new clouds. We have only begun to unravel the processes which determine the mass balance in the multiphase ISM, and it will be a challenge, both theoretical and observational, to improve our understanding.

\subsection{SNR Evolution}

Because this review is focused on the physical processes associated with SNRs in an inhomogeneous medium, we shall provide only a brief list of some of the references which consider the effects of mass exchange and cloud crushing on SNR evolution. Early studies (Chevalier 1975, McKee and Cowie 1975) concentrated on the effects of thermal conduction in young SNRs without, however, allowing for saturation effects. More recently, Hamilton (1985) has analyzed the expansion of a clumpy young SNR under the extreme assumption that the clumps experience no ram pressure deceleration, only ablation. The effects of evaporation on SNRs in their subsequent evolution was first studied by McKee and Ostriker (1977); Chieze and Lazareff (1981) obtained the similarity solution for this problem, and Cowie, McKee, and Ostriker (1981) carried out a numerical simulation. SNR evolution with arbitrary mass injection is discussed by Ostriker and McKee (1987). Clouds also affect SNR evolution by draining momentum from the blast wave due to cloud drag. Cox (1979) was the first to consider such impeded blast waves, under the assumption that the interior is isothermal; Ostriker and McKee (1987) have considered the opposite case of an adiabatic interior. Energy losses due to cloud crushing were considered by Cowie et al. (1981), and analytic solutions have been obtained by Ostriker and McKee (1987). The emission of sound waves due to the interaction of an SNR blast wave with embedded clouds and the consequent heating of the ISM has been discussed by Spitzer (1982) and Spitzer and Ikeuchi (1984).

Despite the fact that SNRs with mass injection are fundamentally different from those without, it has proven difficult to determine whether a given remnant is dominated by mass injection. The primary reason for this sad state of affairs is that most known remnants are about the age of the Cygnus Loop or younger, and for such remnants the observational distinctions between evaporative and Sedov-Taylor remnants are relatively minor (Cowie et al., 1981). Furthermore, the evaporative solution applies to SNRs in a three-phase ISM unaffected by the progenitor star, whereas massive SNRs occur in a radially stratified medium quite different from the normal ISM (see below). Indeed, the Cygnus Loop appears to be such an SNR, and it is no longer tenable to consider it as a possible evaporative remnant, as did Cowie et al. A promising technique for studying mass injection in SNRs is through observations of the infrared emission from collisionally heated dust grains injected into the hot intercloud medium; Dwek (1981) has studied the dust emission expected from evaporating clouds, and more generally one would expect the distribution of dust emission from a remnant dominated by mass injection to be quite different from one that is not. High resolution radio observations provide a good means for studying cloud crushing in SNRs, since most of the radio emission from older remnants is believed to originate from crushed clouds (Blandford and Cowie 1982). 


\section{SNR SHOCKS IN A RADIALLY STRATIFIED ISM}

\subsection{HII Regions and Stellar Wind Bubbles in a Cloudy ISM}

Just as massive stars are the progenitors of supernovae, so, in a sense, the HII regions around these stars are the progenitors of the resulting SNRs. As discussed in the Introduction, the outpouring of energy from a massive star profoundly alters the surrounding medium, converting it from a cloudy medium into one which is radially stratified around the progenitor star. The evolution and appearance of an SNR in such a medium is quite different from that in either a homogeneous medium or a uniform, cloudy medium.

We begin by reviewing the structure of the medium around a massive star embedded in an initially homogeneous medium. The ionizing radiation creates a Strömgren sphere extending out to a radius $R_{i}=66.9\left(S_{49} / n_{i}^{2}\right)^{1 / 3} \mathrm{pc}$, where $n_{i}$ is the density of ionized gas inside $R_{i}$ and $S_{49}$ is the rate of production of ionizing photons in units of $10^{49}$ photons s ${ }^{-1} ; S_{49} \simeq(0.04,1,8)$ for a (B0V, $06.5 \mathrm{~V}, \mathrm{O} 4 \mathrm{~V}$ ) star, respectively (Panagia 1973). The pressure of the ionized gas causes the HII region to expand as $R \propto t^{4 / 7}$ after a time $R_{i} / C_{\Pi}$, where $C_{\Pi}$ is the isothermal sound speed in the ionized gas (Spitzer 1968a). Massive stars have strong stellar winds, with kinetic luminosities $L_{w}=\dot{M}_{w} v_{w}^{2} / 2$ of about $10^{35} \mathrm{erg}$ $\mathrm{s}^{-1}$ for a B0V star and $10^{36} \mathrm{erg} \mathrm{s}^{-1}$ for an O6.5V star (Abbott 1982). Such a wind creates a bubble of hot $\left(T \sim 10^{6} \mathrm{~K}\right)$ gas inside the HII region, and the bubble expands as $R_{b} \propto\left(E / \rho_{0}\right)^{1 / 5} t^{2 / 5} \propto\left(L_{w} / \rho_{0}\right)^{1 / 5} t^{3 / 5}$ (Castor et al. 1975). Because the medium is homogeneous, the radiative losses are small and the bubble can grow quite large by the end of the star's life; e.g., for an O6.5 star, $R_{b} \lesssim 75 n_{m}^{-1 / 5} \mathrm{pc}$, where $n_{m}$ is the ambient density.

The evolution of the ISM around a massive star is quite different if the medium is initially cloudy. The ionizing radiation causes the clouds to lose mass ("photoevaporation"), and since the mass loss is asymmetric, the cloud rockets away from the ionizing star (Oort and Spitzer 1955, Kahn 1969). When ionizing radiation first strikes a cloud, it drives an ionization-shock front into the cloud which crushes the cloud, just as if it were overtaken by an SNR blast wave. Provided the cloud is not too small, the ionized gas flowing away from the cloud has sufficient opacity to shield the cloud from the full brunt of the incident ionizing photons. Balancing ionizations and recombinations in this layer gives $\alpha n_{e}^{2} a \delta=S / 4 \pi r^{2}$, where $\alpha$ is the hydrogen recombination coefficient for excited states and $\delta$ is the fractional thickness of the layer. Calculations show that if $n_{e}$ is measured at the isothermal sonic point and most of the ionizing photons are absorbed in the recombination layer, then $\delta \sim 1 / 6$ (Bertoldi 1987). The ratio of $n_{e}$ to the ambient HII region density $n_{i}$ is then

$$
\frac{n_{e}}{n_{i}}=\left(\frac{R_{i}^{3}}{3 r^{2} a \delta}\right)^{1 / 2}=\frac{774}{r_{\mathrm{pc}} a_{\mathrm{pc}}^{1 / 2}}\left(\frac{S_{49}}{n_{i}^{2}}\right)^{1 / 2} \text {. }
$$

Since the cloud is assumed to lie within the Strömgren radius, this ratio is necessarily greater than unity, provided only that the recombination layer exists. The pressure inside the cloud is $P_{c}=2\left(2 n_{e} k T_{e}\right)$, so that for $T_{e}=10^{4} \mathrm{~K}$,

$$
\frac{P_{c}}{k}=3.1 \times 10^{7} \frac{S_{49}^{1 / 2}}{r_{\mathrm{pc}} a_{\mathrm{pc}}^{1 / 2}} \quad \mathrm{~cm}^{-3} \mathrm{~K}
$$


which, for small clouds close to the star. can be comparable to the pressure inside the Cygnus Loop $\left(P / k \sim 10^{6-7} \mathrm{~cm}^{-3} \mathrm{~K}\right.$ [Raymond et al. 1987]). The crushing of the cloud by the ionization shock front is a radiation-driven implosion, which in principle can drive the cloud into gravitational collapse, thereby inducing star formation (Sandford, Whitaker, and Klein 1982).

Once the cloud has been crushed by the ionization-shock front, it settles into a comet-shaped equilibrium (Bertoldi and McKee 1987), such as that observed for ionized globules in HII regions (Reipurth 1983). The time for the cloud to be destroyed by photoevaporation is $t_{\text {ion }} \simeq 2 a / 3 v_{D}$, where $v_{D}=C_{\mathrm{I}}^{2} / 2 C_{\Pi}$ is the velocity of the ionization front and $C_{\mathrm{I}}^{2}$ is $P / \rho$ in the neutral cloud, including the magnetic pressure. The numerical value of $t_{\text {ion }}$ depends on the structure of the magnetic field in the crushed cloud, since that determines both $C_{\mathrm{I}}^{2}$ and the reduction in the cloud radius $a$. Just as in the case of SNRs, photoevaporating clouds come in several different sizes: tiny clouds are fully ionized before they are crushed; small clouds reach the cometary equilibrium, but are destroyed before the rocket effect can move them very far; medium clouds are displaced a substantial distance before destruction; and large clouds are not crushed before the star dies.

Elmegreen (1976) showed that when a massive star turns on in a cloudy medium, the radius of the Strömgren sphere is initially quite large because the density which determines the radius is that of the intercloud medium. The Strömgren sphere rapidly contracts, however, as the photoevaporated debris from the clouds raises the density in the HII region. Cloud destruction and the rocket effect combine to clear the clouds from a region around the star. McKee et al. (1984) generalized this analysis to the case of a distribution of cloud masses and allowed for cloud crushing. They showed that if the number of clouds more massive than $m$ varies as $1 / m$, then the radius $R_{h}$ of the cleared zone grows as $t^{4 / 7}$, just as does that of a Strömgren sphere in a homogeneous medium. The gas inside $R_{h}$ is homogeneous and has a low density, so it is difficult to see. Much of the photoevaporated gas accretes into an HI shell outside the HII region. If the star is approximately stationary and of spectral type B0 or earlier, then at the end of the star's life the mass of the shell is $M_{h} \sim 2.4 \times 10^{4} n_{m}^{0.1} M_{\odot}$, and its radius is $R_{h} \simeq 56 n_{m}^{-0.3} \mathrm{pc}$, where $n_{m}$ is the mean density of the ambient medium, including clouds. In contrast to an HII region in a homogeneous medium, which is bounded by an ionization front, an HII region in a cloudy medium is bounded by a recombination front because the central star is unable to keep all the photoevaporated gas ionized (Shull et al. 1985). The presence of clouds in the HII region dramatically alters the evolution of the stellar wind bubble, since photoevaporated gas causes catastrophic cooling if the bubble attempts to expand beyond the homogenization radius $R_{h}$ (McKee et al. 1984); as a result, a stellar wind bubble is regulated so that it is intermediate between an adiabatic bubble (Castor et al. 1975) and a momentum-conserving one (Avedisova 1972; Steigman, Strittmatter and Williams 1975). The resulting picture of the evolution of an HII region is consistent with Chu's (1981) and Lozinskaya's (1982) classification of nebulae around Wolf-Rayet stars and Of stars. 


\subsection{Massive SNRs}

When an isolated, massive star explodes, we expect the ambient medium to consist of one or more shells of circumstellar material close to the star; next, a very low density $\left(n \sim 10^{-2}-10^{-3} \mathrm{~cm}^{-3}\right)$ stellar wind bubble $\left(R<R_{b}\right)$; then a region of low density, homogenized HII $\left(R_{b}<R<R_{h}\right)$; a region of HII in which large clouds survive $\left(R_{h}<R<R_{i}\right)$; and finally a dense HI shell at $R \sim R_{i}$. Even in the absence of a stellar wind, the fact that the ionizing radiation from the star homogenizes the ambient medium out to a radius $R_{h} \simeq 56 n_{m}^{-0.3} \mathrm{pc}$ (for $M \gtrsim 20 M_{\odot}$ ) implies that any dense gas within $R \sim 20 \mathrm{pc}$ of the star at the end of its life must be circumstellar (i.e., ejected from the star) rather than interstellar; in particular, much of the nebulosity that Chu (1981) observed around Wolf-Rayet stars must be circumstellar (McCray 1983; McKee et al. 1984). The details of the evolution of interstellar bubbles in this environment remain to be worked out, but it is likely that the bubbles around massive stars $\left(M \gtrsim 20 M_{\odot}\right)$ will extend out to $\gtrsim 20 \mathrm{pc}$ as well. We conclude that young, massive SNRs interact primarily with circumstellar material. This implies that the dust to gas ratio in these objects may not be standard. Lozinskaya (private communication, 1987) has pointed out that the difference between "naked" and "clothed" Crabs may simply reflect differences in the timing and amount of circumstellar mass ejection.

While the SNR blast wave is interacting with the circumstellar matter, it will deviate from free expansion $\left(v_{b}=\right.$ const.), but unless the circumstellar mass $M_{c i r}$ greatly exceeds the mass $M_{e j}$ ejected by the supernova, it will not enter the Sedov-Taylor stage. Once the blast wave enters the stellar wind cavity, it will return to free expansion. Hence, young, massive SNRs remain in free expansion out to

$$
R_{A} \simeq 2.1\left(\frac{M_{e j}+M_{c i r}}{M_{\odot} n}\right)^{1 / 3} \quad \mathrm{pc},
$$

which for $M_{e j}+M_{\text {cir }}=10 M_{\odot}$ and $n=10^{-2} \mathrm{~cm}^{-3}$ is $20 \mathrm{pc}$. The low density in the bubble and in the surrounding homogenized HII region reduce the luminosity of the SNR below the value it would otherwise have had: massive SNRs are muffled. For example, if the wind bubble extended out to $20 \mathrm{pc}$, beyond which the density jumped to $n=1 \mathrm{~cm}^{-3}$, then the SNR would never be bright in X-rays after the emission from the shocked ejecta and circumstellar matter faded out: the density inside the bubble is too low to give detectable $\mathrm{X}$-ray emission, and after the blast wave strikes the shell the post-shock temperature would be less than $10^{6} \mathrm{~K}$. Note that all three effects discussed here-the circumstellar interaction, the free expansion, and the muffling-also apply to the large number of massive SNRs which occur in stellar associations. These effects can be reduced if the star is moving through a relatively high density medium so that the Strömgren radius is small: in the direction of motion of the star, the Strömgren radius is reduced to $R_{i}=5.8 /\left(n_{m 2}^{2} v_{* 6}^{4}\right)^{1 / 3} \mathrm{pc}$, where $v_{* 6}$ is the stellar velocity in units of $10^{6} \mathrm{~cm}$ $\mathrm{s}^{-1}$ and $n_{m 2}=n_{m} /\left(10^{2} \mathrm{~cm}^{-3}\right)$.

The observed morphology of optical filaments in SNRs such as the Cygnus Loop is a problem of long standing: if the blast wave is interacting with interstellar clouds, then one would expect SNRs to show a scalloped appearance as shocks wrap around the clouds (McKee and Cowie 1975; Tenorio-Tagle and Rozyczka 1986; Rozyczka and Tenorio-Tagle 1987), whereas in fact the filaments 
are curved in the opposite sense and tend to follow the curvature of the blast wave. This problem led McKee and Cowie (1975) to suggest that the clouds near the Cygnus Loop are smaller than the thickness of the shell $(a<1 \mathrm{pc})$, so that the scalloped appearance would occur only on small scales, but careful observations (Hester, Parker, and Dufour 1983) show that this interpretation is no longer tenable. Instead, the blast wave appears to be interacting with large, roughly spherical sheets, just as expected for an SNR in a medium which has been radially stratified by its progenitor star. McCray and Snow (1979) suggested that the wind from the progenitor would lead to this result, and we now see that the effects of photoionization are at least as important. Careful studies of the optical morphology of other middle-aged SNRs would be quite valuable.

Our discussion has concentrated on SNRs from massive progenitors $\left(M \gtrsim 20 M_{\odot}\right.$ ) because such SNRs spend their entire observable life interacting with either circumstellar matter or interstellar matter that has been processed by the progenitor. However, the number of SNRs from lower mass B stars is expected to be comparable to that from more massive stars. Their behavior will resemble that of their more massive cousins so long as the blast wave radius is less than $R_{i}$, and then will gradually approach that of Type Ia SNRs. The Cygnus Loop is a likely example: Charles et al. (1985) have argued that the absence of clouds embedded in the Loop implies that it is the remnant of a high mass star, and that the presence of the filaments within $20 \mathrm{pc}$ of the center limits the spectral type of the progenitor to later than B0. Two examples have been suggested in the LMC: N49 (Shull et al. 1985) and N132D (Hughes 1987). Shull et al. have developed a detailed model for N49 based on the hypothesis that the progenitor was a B star which processed the ambient cloudy medium by photoionization. They point out the pressure in the HII region dropped when the B star evolved into a red supergiant, allowing the inner edge of the HI shell to encroach into the HII cavity, and they suggest that this effect is apparent in the X-ray data.

\section{CONCLUSIONS}

The inhomogeneous structure observed in the ISM is due in no small part to SNRs, and observations of SNRs provide a potentially powerful probe of this structure. Supernovae from massive progenitor stars $\left(M \gtrsim 20 M_{\odot}\right)$, including both Type II and probably Type Ib, occur in a medium which has been completely transformed by mass ejection and energy injection from the progenitor, and the resulting remnants therefore tell us more about stellar evolution than about the ISM. In their youth, massive SNRs are most readily observed while interacting with circumstellar material. Stellar wind bubbles allow such SNRs to remain in approximate free expansion out to relatively large radii. Once they expand beyond the circumstellar material, they are "muffled" by the low density in the wind bubble and homogenized HII region; this can be accentuated in stellar associations. Even in old age, such SNRs interact primarily with matter processed by their progenitor stars, unless a high progenitor velocity or a nearby dense molecular cloud permits ambient interstellar matter to lie near the supernova. SNRs from lower mass progenitors-corresponding to both Type Ia SN and Type II SN from B stars of intermediate mass - can probe the structure of the "pristine" ISM, but we are only beginning to unravel the complex physical processes associated with the interaction of a blast wave with an interstellar cloud, and to distinguish such remnants from their more massive counterparts. 
Acknowledgments. I wish to thank Sam Falle for several provocative remarks, and Frank Bertoldi and Denis Cioffi for comments on the manuscript. This research is supported in part through NSF grant 86-15177.

\section{REFERENCES}

Abbott, D.C. 1982, Ap.J., 263, 723.

Avedisova, V.S. 1972, Sov. Astron. A.J., 15, 708.

Balbus, S.A. 1985, Ap.J., 291, 518.

-. 1986, Ap.J., 304, 787.

Balbus, S.A., and McKee, C.F. 1982, Ap.J., 252, 529.

Ballet, J., Arnaud, M., and Rothenflug, R. 1986, Astron. Astrophys., 161, 12.

Batchelor, G. 1967, An Introduction to Fluid Dynamics, (Cambridge: Cambridge University Press).

Begelman, M.C., and McKee, C.F. 1987, in preparation.

Bertoldi, F. 1987, in preparation.

Bertoldi, F., Klein, R., McKee, C.F., and Sandford, M.T. 1987, in preparation.

Bertoldi, F. and McKee, C.F. 1987,in preparation.

Blandford, R.D., and Cowie, L.L. 1982, Ap.J., 260, 625.

Böhringer, H., and Hartquist, T. 1987, Astron. Astrophys., (submitted).

Bychkov, K.V., and Pikel'ner 1975, Sov. Astr. Letters, 1, 14.

Campbell, P.M. 1984, Phys. Rev. A., 30, 365.

Castor, J., McCray, R., and Weaver, R. 1975, Ap.J. (Letters), 200, L107.

Charles, P., Kahn, S., and McKee, C.F. 1985, Ap.J., 295, 456.

Chernyi, G.G. 1961, Introduction to Hypersonic Flow, (NY: Academic Press).

Chevalier, R.A. 1975, Ap.J., 200, 698.

Chieze, J.P., and Lazareff, B. 1981, Astron. Astrophys., 95, 194.

Chiosi, C., and Maeder, A. 1986, Ann Rev. Astron. Astrophys., 24, 329.

Chu, Y.H. 1981, Ap.J., 249, 195.

Cowie, L.L., and McKee, C.F. 1977, Ap.J., 211, 135.

Cowie, L.L., McKee, C.F., and Ostriker, J.P. 1981, Ap.J., 247, 908.

Cowie, L.L., and Songaila, A. 1977, Nature, 266, 501.

Cox, D.P. 1979, Ap.J., 234, 863.

. 1987, this volume.

Cox, D.P., and Smith, B.W. 1974, Ap.J. (Letters), 189, L105.

de Jong, T. 1980, in Highlights of Astronomy Vol. 5, ed. P.A. Wayman (Reidel: Dordrecht), p. 301.

Doroshkevich, A.G., and Zel'dovich, Ya. B. 1981, Sov. Phys. J.E.T.P., 53, 405.

Draine, B.T., and Giuliani, J.L. 1984, Ap.J., 281, 690.

Dwek, E. 1981, Ap.J., 246, 430.

Elmegreen, B.G. 1976, Ap.J., 205, 405.

Field, G.B. 1965, Ap.J., 142, 531.

Giuliani, J.L. 1984, Ap.J., 277, 605.

Graham, R., and Langer, W.D. 1973, Ap.J., 179, 469.

Hamilton, A.J.S. 1985, Ap.J., 291, 513.

Heathcote, S.R., and Brand, P.W.J.L. 1983, M.N.R.A.S., 203, 67.

Hester, J.J., Parker, R.A.R., and Dufour, R.J. 1983, Ap.J., 273, 219.

Hollweg, J.V. 1986, Ap.J., 306, 730.

Hughes, J.P. 1987, Ap.J., 314, 103.

Ikeuchi, S., Habe, A., and Tanaka, Y.D. 1984, M.N.R.A.S., 207, 909.

Kahn, F.D. 1969, Physica, 41, 172.

-_. 1976, Astron. Astrophys., 50, 145.

Krebs, J., and Hillebrandt,.W. 1983, Astron. Astrophys., 128, 41. 
Landau, L.D., and Lifschitz, E.M. 1959, Fluid Mechanics, (Reading: Addison Wesley).

Lozinskaya, T.A. $1982, A p$. and $S p$. Sci., 87, 313.

Luciani, J.F., Mora, P., and Pellat, R. 1985, Phys. Fluids, 28, 835.

Max, C.E., McKee, C.F., and Mead, W.C. 1980, Phys. Fluids, 23, 1620.

McCray, R. 1983, Highlights of Astronomy, 6, 565.

McCray, R., and Snow, T.P. 1979, Ann. Rev. Astron. Astrophys., 17, 213-40.

McCray, R., Stein, R.F., and Kafatos, M. 1975, Ap.J., 196, 565-70.

McKee, C.F. 1982, in Supernovae: A Survey of Current Research, M.J. Rees and R.J. Stoneham (eds.), (Reidel: Dordrecht, Holland), p. 433.

- 1986, Astrophys. and Sp. Sci., 118, 383.

McKee, C.F., and Cowie, L.L. 1975, Ap.J., 195, 715.

-. 1977, Ap.J., 215, 213-25.

McKee, C.F., Cowie, L.L., and Ostriker, J.P. 1978, Ap.J. (Letters), 219, L23.

McKee, C.F., Hollenbach, D., Seab, C., Tielens, A. 1987, Ap.J., (in press).

McKee, C.F., and Ostriker, J.P. 1977, Ap.J., 218, 148.

McKee, C.F., Van Buren, D., and Lazareff, B. 1984, Ap.J. (Letters), 278, L115.

Nittman, J. 1981, M.N.R.A.S., 197, 699.

Nittman, J., Falle, S., and Gaskell, P. 1982, M.N.R.A.S., 201, 833.

Nulsen, P.E.J. 1982, M.N.R.A.S., 198, 1007.

Oettl, R., Hillebrandt, W., and Müller, E. 1985, Astron. Astrophys., 151, 33.

Oort, J.H., and Spitzer, L. 1955, Ap.J., 121, 6.

Ostriker, J.P., and McKee, C.F. 1987, Rev. Mod. Phys. (submitted).

Panagia, N. 1973, Astron. J., 78, 929.

Raymond, J.C., Hester, J.J., Cox, D., Blair, W.P., Fesen, R.A., and Gull, T.R. 1987, Ap.J., submitted.

Reipurth, B. 1983, Astron. Astrophys., 117, 183.

Richtmyer, R.D. 1960, Com. Pure Appl. Math, 13, 297.

Rozyczka, M., and Tenorio-Tagle, G. 1987, Astron. Astrophys., 176, 329.

Sandford, M.T., Whitaker, R.W., and Klein, R.J. 1982, Ap.J., 260, 183.

Sgro, A.G. 1975, Ap.J., 197, 62l.

Shull, P., Dyson, J.E., Kahn, F.D., and West, K. 1985, M.N.R.A.S., 212, 799.

Silk, J., and Solinger, A. 1973, Nature, 244, 101.

Smith, D.F. 1986, Ap.J., 302, 836.

Spitzer, L. 1962, Physics of Fully Ionized Gases, (New York: Wiley).

—. 1968a, Diffuse Matter in Space, (New York: Wiley).

- $1968 \mathrm{~b}$, in Stars and Stellar Systems VII: Nebulae and Interstellar Matter ed. B. Middlehurst and L. Aller (Chicago: U. Chicago Press), p. 1. 1982, Ap.J., $262,315$.

Spitzer, L., and Ikeuchi, S. 1984, Ap.J., 283, 825.

Steigman, G., Strittmatter, P.A., and Williams, R.E. 1975, Ap.J., 198, 575.

Tenorio-Tagle, G., and Rozyczka, M. 1986, Astron. Astrophys., 155, 120.

Wheeler, J.C., and Levreault, R. 1985, Ap.J. (Letters), 294, L17.

Woodward, P.R. 1976, Ap.J., 207, 484.

-. 1979, in IAU Symp. 84, The Large Scale Character of the Galaxy, ed. W.B. Burton (Dordrecht: Reidel), p. 159. 